\title{
Formation of Au Microbump Arrays for Flip-Chip Bonding Using Electroless Au Deposition from a Non-Cyanide Plating Bath
}

\author{
Tokihiko Yokoshima*, Kentaro Nomura*,**, Yasuhiro Yamaji*, Katsuya Kikuchi*, Hiroshi Nakagawa*, \\ Kohji Koshiji**, Masahiro Aoyagi*,**, Ryota Iwai***, Tomoaki Tokuhisa*** and Masaru Kato*** \\ *National Institute of Advanced Industrial Science and Technology (AIST), Tsukuba Central 2, 1-1-1 Umezono, Tsukuba, Ibaraki, 305-8568, Japan \\ **Graduate School of Science and Technology, Tokyo University of Science, 2641 Yamazaki, Noda, Chiba, 278-8510, Japan \\ ***Kanto Chemical Co., Inc., 1-7-1 Inari, Soka, Saitama, 340-0003, Japan
}

(Received August 11, 2009; accepted November 2, 2009)

\begin{abstract}
The formation of Au microbump arrays for flip-chip bonding was investigated using an electroless Au plating bath. Generally, electroless Au deposition gives a low deposition rate, preventing the fabrication of Au bumps with heights of more than $5 \mu \mathrm{m}$. To overcome this problem, we developed a new electroless Au deposition process that employs a non-cyanide bath. This process delivers a high deposition rate, especially for fine bump patterns (bumps of less than $10 \mu \mathrm{m}$ in diameter). Au bumps with a diameter and height of $10 \mu \mathrm{m}$ each could be fabricated with a high deposition rate using the new electroless Au plating bath. Moreover, Au microbump arrays were successfully fabricated on $\mathrm{Cu}$ wiring. The practical feasibility of this technique was demonstrated by forming arrays with bump heights and bump diameters of 10 $\mu \mathrm{m}$ each and an array pitch of $30 \mu \mathrm{m}$. All film-formation processes were performed under cyanide-free conditions; thus, this process has a high industrial potential for fabricating ultra-fine Au microbump arrays.
\end{abstract}

Keywords: Electroless Au Deposition, Non-Cyanide Bath, Flip-Chip Bonding, Bump

\section{Introduction}

Interconnect technologies, such as chip-to-chip and chip-to-substrate, are very important for fabricating highperformance electrical devices.[1] To achieve the required performance, high-density interconnects are necessary, and flip-chip bonding is one of the best methods for achieving such interconnects. In recent years, interconnect density has increased and bump sizes for first-level interconnects have decreased to less than $10 \mu \mathrm{m}$. Usually, microbumps and solder-bumps are used for flip-chip bonding of chip-to-substrate interconnects.[2-4] When using solder-bumps for interconnects, the bump shape is affected by the reflow process, and decreasing the bump pitch is not easy. On the other hand, it is easy to attain a high-density pad pitch of microbumps, though obtaining bump arrays with a uniform bump height is essential. Au bumps are usually fabricated by electrodeposition. However, due to current distribution, it is not easy to fabricate homogeneous $\mathrm{Au}$ bumps with fine pitch (e.g., $<20 \mu \mathrm{m}$ ). Moreover, lead wires supplying the current for Au electrodeposition disturb high-speed signal transmissions.[5]
In this situation, electroless $\mathrm{Au}$ deposition is one of the best methods for obtaining fine-pitch Au bumps. The electroless deposition method requires no electrical lead wiring and is not affected by the current distribution. However, conventional electroless Au deposition with high deposition rate is currently feasible only if a cyanide bath is used.[6] With this technique, it is difficult to obtain fine structures because the photoresist pattern and/or the substrate are damaged by cyan ions during the deposition. On the other hand, the deposition rate of a non-cyanide bath is too low to fabricate Au bumps with heights of more than $5 \mu \mathrm{m}$.[6] In addition, a cyanide bath is not desirable from an environmental viewpoint.

In this study, Au microbump arrays with a bump diameter and bump height of $10 \mu \mathrm{m}$ each (aspect ratio $=1$ ) were fabricated using a newly developed non-cyanide bath. Flipchip bonding experiments were carried out and some bonding parameters were investigated. Adhesive/barrier layers were developed using an electroless deposition process, and the development and characterization of the $\mathrm{Au}$ bump arrays on test chips with wiring was also investigated. 


\section{Experimental}

Low temperature, neutral $\mathrm{pH}$, and cyanide-free conditions are needed for micro-bump formation, because the resist pattern is easily damaged by high temperature, alkaline $\mathrm{pH}$, and/or cyanide. In this investigation, the bath system was based on the electroless $\mathrm{Au}$ plating bath "SELFGOLD OTK" (Kanto Chemical Inc.) because this bath is a non-cyanide Au plating bath. Gold(I) trisodium disulphite was used as the metal source with a standard $\mathrm{Au}$ concentration was of $2 \mathrm{~g} \mathrm{dm}^{-3}$. Mixed ligand system was used for Au-complex and both sulfite and thiosulfate ions were used as complexing agent. Some surfactants and stabilizers were used to improve the stability of the plating bath. The bath $\mathrm{pH}$ was 7.2 , and the bath temperature was $60^{\circ} \mathrm{C}$. However, using this commercial bath, micro-bumps could not be fabricated because the resist patterns were damaged during immersion in the plating bath. A new plating bath is needed based on this commercial bath. For basic investigations, such as those on deposition behavior and die share test, an evaporated $\mathrm{Au}(100 \mathrm{~nm}) / \mathrm{Ti}(20 \mathrm{~nm}) /$ Si wafer (3 in.) was used as the substrate. For advanced investigations, such as those on the formation of barrier layers and the electrical continuity test, an evaporated $\mathrm{Cu}$ $(100 \mathrm{~nm}) / \mathrm{Ti}(10 \mathrm{~nm}) / \mathrm{Si}$ wafer (3 in.) was used as the substrate. A resist pattern with a diameter of 5-20 $\mu \mathrm{m}$, pitch of $10-30 \mu \mathrm{m}$, and thickness of about $12 \mu \mathrm{m}$ was made using photolithography with photoresist (PMER PLA900PM). Cu wires with a thickness of $3 \mu \mathrm{m}$ were fabricated by a semi-additive process using $\mathrm{Cu}$ electrodeposition, chemical etching, and reactive ion etching. The barrier layers used for Ti evaporation and electroless $\mathrm{NiB}$ deposition were fabricated using the lift-off process and the semi-additive method, respectively. Details of the wiring and the barrier layer are discussed below in the Results and Discussion section. The test chip and the substrate were cut to dimensions of $10 \times 10 \mathrm{~mm}^{2}$ and $15 \times 15 \mathrm{~mm}^{2}$, respectively. Flip-chip bonding experiments employed a high-precision flip-chip bonder. Bonding tests were carried out using a die share experiment. The shape of the bumps was observed using an optical microscope, laser microscope, and scanning electron microscope (SEM).

\section{Results and Discussion}

\subsection{Electroless Au plating bath with high deposition rate}

In general, the rate of electroless Au deposition using a non-cyanide bath is lower than that using a cyanide bath.[6] Figure 1 shows the deposition rate of some electroless $\mathrm{Au}$ deposition techniques. To form bump arrays with heights of more than $5 \mu \mathrm{m}$, a deposition rate of 3.5 $\mu \mathrm{m} / \mathrm{h}$ is necessary to avoid damaging the photoresist during deposition given a bath with a $\mathrm{pH}$ of about 7 and a temperature of $50^{\circ} \mathrm{C}$. Conventional techniques to increase the deposition rate consist of increasing the bath $\mathrm{pH}$ and/or the bath temperature. However, this results in greater damage to the photoresist pattern. Another technique consists of increasing the $\mathrm{Au}$ ion and/or the reducing agent concentration, but this technique leads to a decrease in stability. The addition of chemical agents such as an accelerator is very effective in increasing the deposition rate, but

\begin{tabular}{|c|c|c|c|c|c|c|}
\hline \multirow[t]{2}{*}{ Bath system } & \multirow[t]{2}{*}{ Bump diameter $(\mu \mathrm{m})$} & \multicolumn{5}{|c|}{ Deposition rate $(\mu \mathrm{m} / \mathrm{h})$} \\
\hline & & 0 & 2 & $4 \quad 6$ & 6 & 8 \\
\hline Common cyanide bath & non patterned & & & & & \\
\hline Common non-cyanide bath & non patterned & $\Delta$ & & & & \\
\hline High-rate non-cyanide bath & non patterned & & & & & \\
\hline SELF GOLD OTK & non patterned & 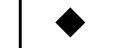 & & & & \\
\hline Electroless Au plating bath $\mathrm{K}$ & non patterned & 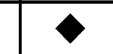 & & & & \\
\hline Electroless Au plating bath $\mathrm{K}$ & 10 & & $>$ & & & \\
\hline Electroless Au plating bath $\mathrm{K}$ & 5 & & & $>$ & & \\
\hline Electroless Au plating bath XG & non patterned & 0 & & & & \\
\hline Electroless Au plating bath XG series & 20 & & 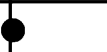 & & & \\
\hline Electroless Au plating bath XG series & 10 & & , & - & -0 & \\
\hline Electroless Au plating bath XG series & 5 & & O- & & -0 & \\
\hline & & 0 & 2 & 4 & 6 & 8 \\
\hline
\end{tabular}

Fig. 1 Deposition rate of several electroless Au deposition baths on patterned and non-patterned substrates. The filled symbols indicate the results when using optimized conditions. 
also leads to modifications in film properties. Thus, we investigated fabricating Au microbumps using a noncyanide plating bath with a high deposition rate.

First, the acceleration of the cathodic reaction was investigated. The deposition rate of electroless plating is strongly related to the rate-determining step. The deposition rate of the basic plating bath, "SELFGOLD OTK," is related to the $\mathrm{Au}$ ion concentration, indicating that the rate-determining step is the mass transfer of $\mathrm{Au}$ ions. Thus, the metal ion concentration was increased from $2 \mathrm{~g}$ $\mathrm{dm}^{-3}$ to $4 \mathrm{~g} \mathrm{dm}^{-3}$. The plating bath was stabilized by decreasing the bath temperature from $60^{\circ} \mathrm{C}$ to $50^{\circ} \mathrm{C}$. Moreover, a high concentration of Au ions near the deposition surface was obtained to decrease the deposition area, leading to the nonlinear diffusion of Au ions. A small-diameter bump array pattern is expected to increase the deposition rate. The data of Fig. 1 show that the deposition rate increases with a decrease in the bump diameter (see Fig. 1; row title: electroless Au plating bath K). Using a pattern with a bump diameter of $10 \mu \mathrm{m}$, we obtained a deposition rate of $3.5 \mu \mathrm{m} / \mathrm{h}$. Decreasing the diameter to $5 \mu \mathrm{m}$ increased the deposition rate to $5 \mu \mathrm{m} / \mathrm{h}$. Thus, this method is very effective for increasing the deposition rate. From these results, both a high deposition rate and bath stability were realized with a high Au concentration of $4 \mathrm{~g}$ $\mathrm{dm}^{-3}$ and low bath temperature of $50^{\circ} \mathrm{C}$. The low bath temperature was also utilized to avoid damaging the photoresist during deposition. Hereafter, this bath, having a high deposition rate, is referred to as the "electroless Au plating bath K" (bath K).

Figure 2 shows the Vickers hardness of the deposited non-patterned films. In this research, we used both the SELFGOLD OTK bath and Bath K, and an annealing tem-

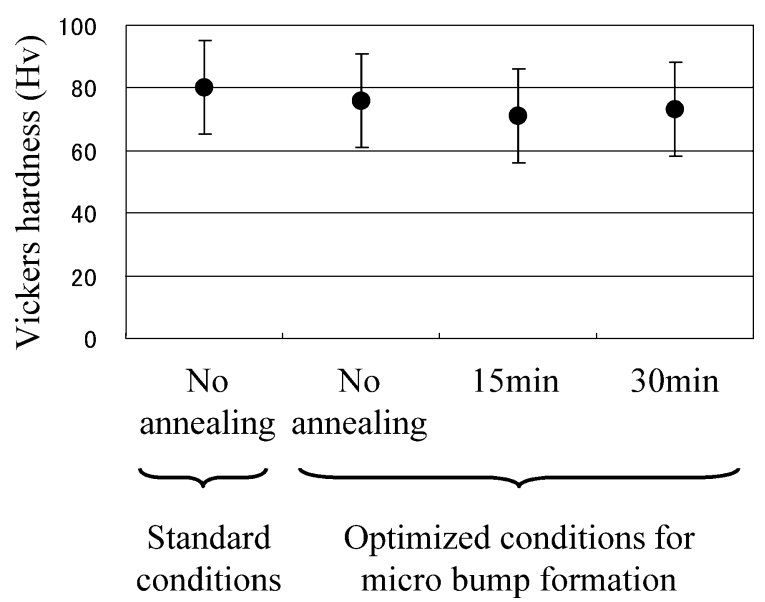

Fig. 2 Vickers hardness of non-patterned Au films deposited by electroless deposition with various annealing times. perature of $250^{\circ} \mathrm{C}$. The film thickness was about $10 \mu \mathrm{m}$. The results showed a good Vickers hardness of less than $80 \mathrm{Hv}$ both with and without annealing, which is the same value obtained from the films fabricated using the SELFGOLD OTK bath. This indicates that the Au bumps fabricated by electroless deposition are suitable for forming fine bump arrays. However, the deposition rate was easily modified by agitation or by using different deposition conditions. Improving the reproducibility of this method, however, is necessary before this plating bath can be used for practical applications.

Next, some additives were introduced into Bath $\mathrm{K}$ in order to increase the deposition rate. The concentrations of surfactants and stabilizers were optimized for use with the accelerator. As seen in Fig. 1, the deposition rate increased with a decrease in the bump diameter (see Fig. 1; row title: Electroless Au plating Bath XG). The filled symbols in Fig. 1 indicate the results when using optimized conditions (note, especially, that the deposition rate for the 10- $\mu$ m-diameter bumps increases). In addition, the stability of the plating bath is excellent, so that no deposition occurs on a non-patterned section of the substrate under optimized accelerator concentration conditions. Thus, this bath series proves very effective for fabricating fine bump arrays with bump diameters of $10 \mu \mathrm{m}$. In spite of the high Au concentration with this plating solution, the plating bath showed excellent stability. So it is likely to have a bath-life as long as that of a conventional, noncyanide bath for electroless Au plating. The volume of Au deposits in the arrays was decreased because of decreasing both the diameter and the height of the bump. Thus, this result indicates decreased costs for micro-bump formation. From these results, both high deposition rate and high bath stability were realized by using a high Au concentration, low bath temperature, and additives. Hereafter, this bath, with the optimized accelerator concentration, will be referred to as the "Electroless Au-Plating Bath XG." (Bath XG).

\subsection{Fabrication of Au bumps with a high aspect ratio}

We developed a process to fabricate Au bump arrays with bump diameters of $10 \mu \mathrm{m}$, bump heights of $10 \mu \mathrm{m}$, and an array pitch of $30 \mu \mathrm{m}$ using Bath XG. In a peripheral array, the number of bumps is about 1100 . Figure 3 shows SEM images and a cross-sectional profile of a Au bump array fabricated by electroless deposition. These images show that bumps with a high aspect ratio $(\fallingdotseq 1)$ are successfully formed when using a fine photoresist pattern. 


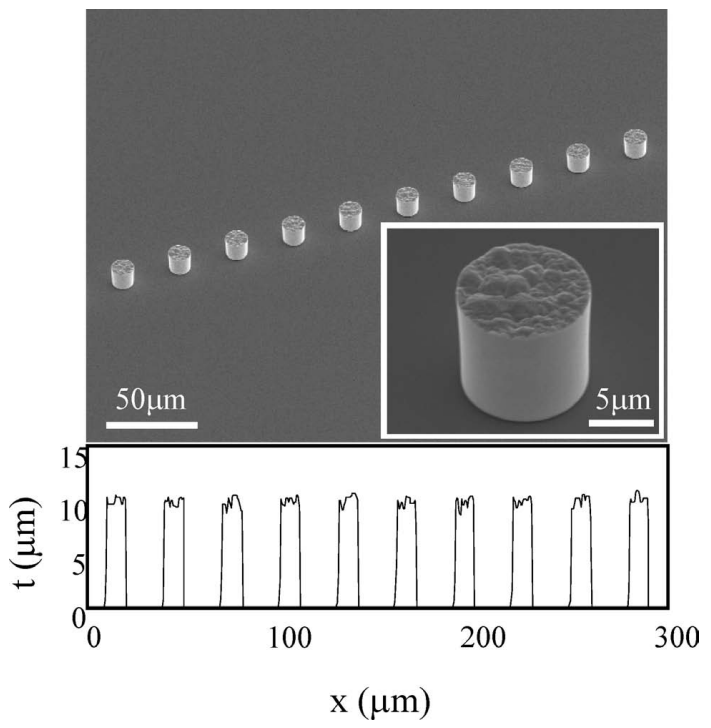

Fig. 3 SEM images and a cross-sectional profile of a $\mathrm{Au}$ bump array fabricated by electroless deposition. The bump diameter, bump height, and array pitch are $10 \mu \mathrm{m}, 10 \mu \mathrm{m}$, and $30 \mu \mathrm{m}$, respectively. The analysis areas differ.

Observation of deposition failure was checked by optical microscope, although a no-deposition failure was not apparent.

Furthermore, we also fabricated bump arrays with bump diameters of $5 \mu \mathrm{m}, 10 \mu \mathrm{m}$, and $20 \mu \mathrm{m}$, as shown in Fig. 4 . The bump heights were controlled by adjusting the deposition time. The figure shows the fabrication of smoothwalled bumps with diameters of 5-20 $\mu \mathrm{m}$. In particular, 5 - $\mu$ m-diameter bumps had a high aspect ratio of about 2 , and with no defects. The deposition reaction of this electroless Au plating bath did not generate hydrogen bubbles, so Au bumps with no defects could be easily obtained without highly controlled agitation systems.

\subsection{Fabrication of Au bumps in fine-pitch arrays}

Au bump arrays with bump:space $=1: 1$ were fabricated to realize a finer bump pitch. In this research, Bath $\mathrm{K}$ was used, with a fixed deposition time of $90 \mathrm{~min}$. Figure 5 shows SEM images of bump arrays with fine pitches of 20 $\mu \mathrm{m}$ and $10 \mu \mathrm{m}$. The bump arrays with diameters of $5 \mu \mathrm{m}$ and $10 \mu \mathrm{m}$ have pitches of $10 \mu \mathrm{m}$ and $20 \mu \mathrm{m}$ and heights of $5 \mu \mathrm{m}$ and $7 \mu \mathrm{m}$, respectively. We analyzed the bump height deviations before dicing the chip and found deviations for the 5 - and 10- $\mu \mathrm{m}$-diameter bumps to be $\pm 0.2 \mu \mathrm{m}$ and $\pm 0.1 \mu \mathrm{m}$ from average height, respectively. We conclude from these results that electroless Au deposition is suitable for fabricating high-density bump arrays with low height deviations.
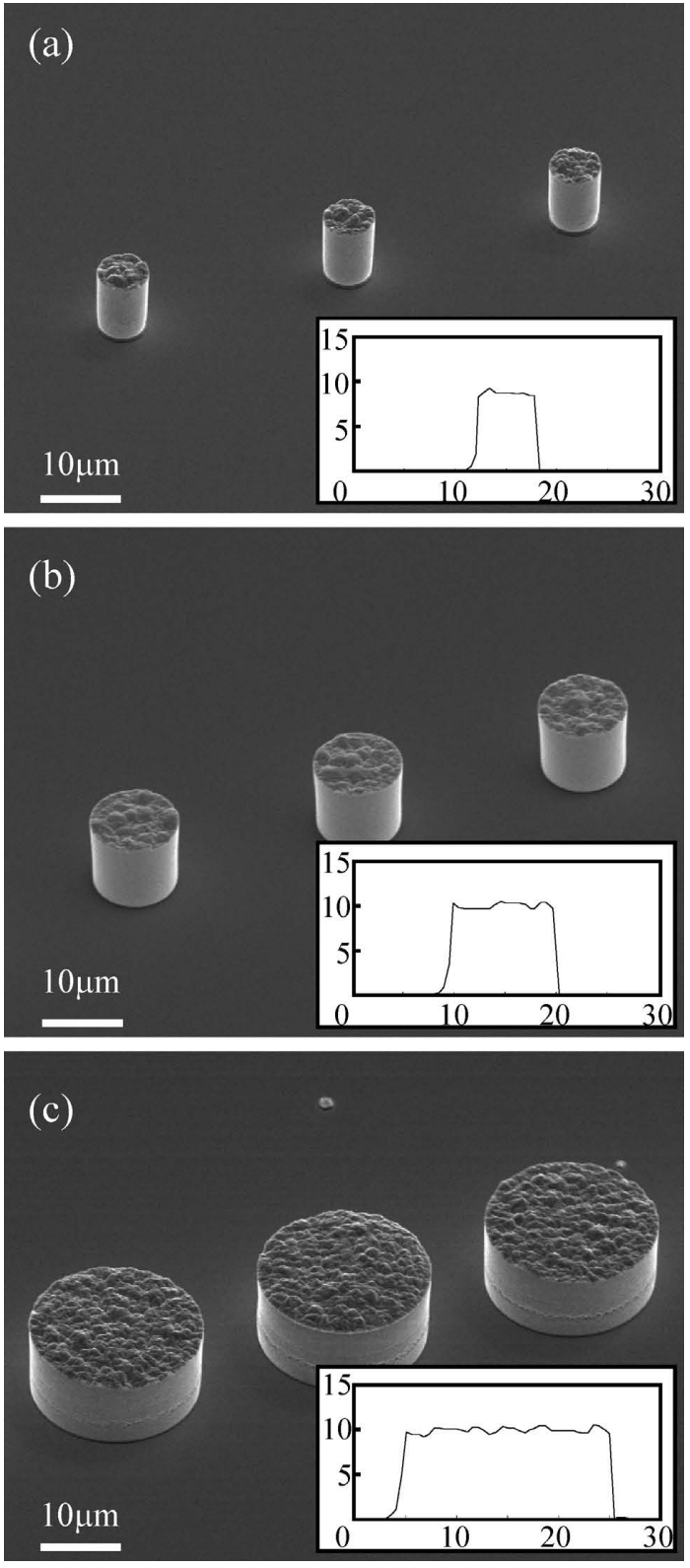

Fig. 4 SEM images of Au bump arrays with bump diameters of (a) $5 \mu \mathrm{m}$, (b) $10 \mu \mathrm{m}$, and (c) $20 \mu \mathrm{m}$. The inset shows a cross-sectional profile of a Au bump fabricated by electroless deposition.

\subsection{Bondability of fine Au bump arrays}

To investigate the bondability of the Au bump arrays, we fabricated chips from the Au bump arrays with a bump diameter of $10 \mu \mathrm{m}$, bump height of $5 \mu \mathrm{m}$, and array pitch of $20 \mu \mathrm{m}$. A chip size of $5 \mathrm{~mm} \times 5 \mathrm{~mm}$ was used. The chip was mounted on a Au $(1.5-2.0 \mu \mathrm{m}) / \mathrm{Au}(100 \mathrm{~nm}) / \mathrm{Ti}(20$ $\mathrm{nm}) / \mathrm{Si}$ substrate. Non-patterned Au films with thicknesses of 1.5-2.0 $\mu \mathrm{m}$ were prepared from Bath $\mathrm{K}$ by electroless Au deposition. Before flip-chip bonding, the surface of the substrate and the Au bumps on the chips were cleaned by $\mathrm{O}_{2}$ plasma ashing. 
Figure 6 shows cross-sectional SEM images of the chip on the substrate after flip-chip bonding. As the images indicate, the Au bumps on the chip and the Au layer on the

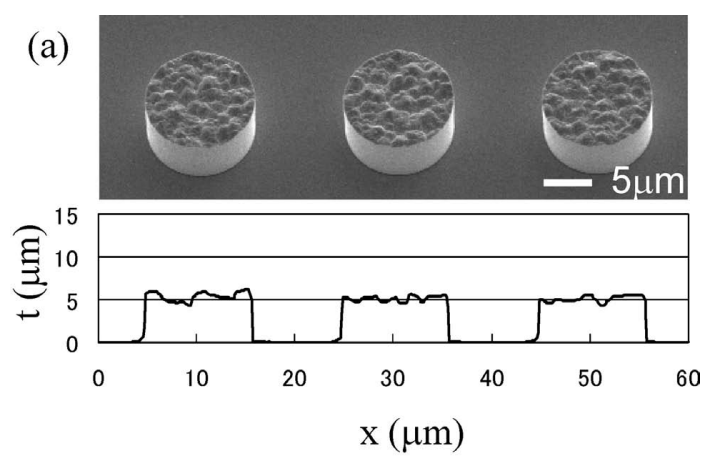

(b)
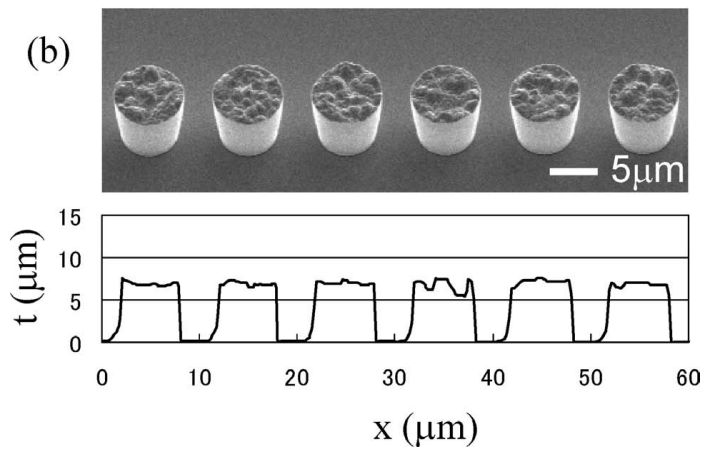

Fig. 5 SEM images and cross-sectional profiles of Au bump arrays with pitches of (a) $20 \mu \mathrm{m}$ and (b) $10 \mu \mathrm{m}$ fabricated by electroless deposition.

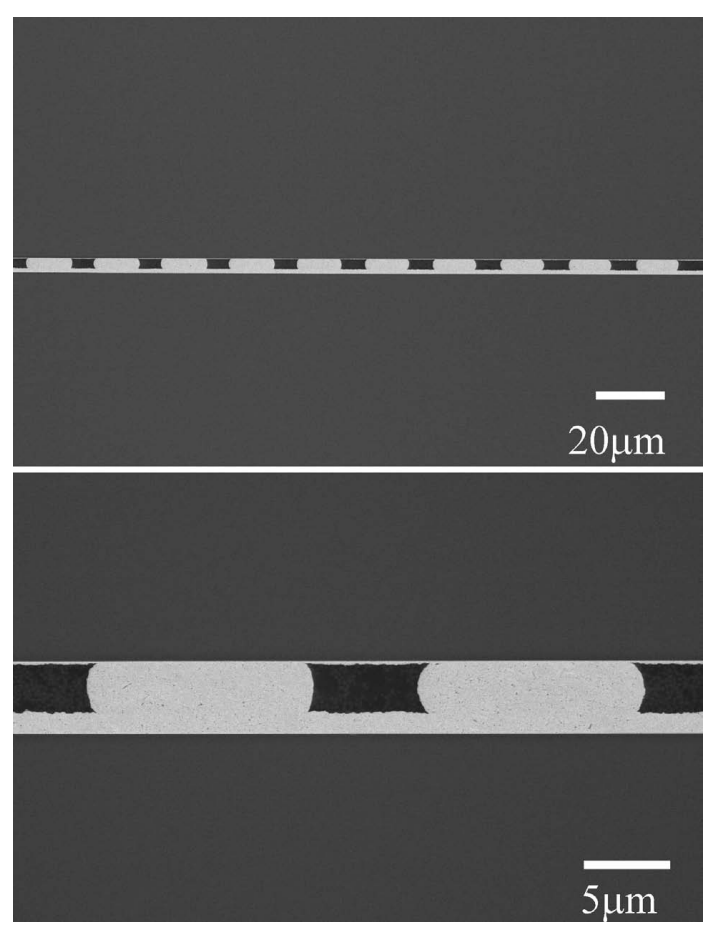

Fig. 6 Cross-sectional SEM images of the test chip on the substrate after flip-chip bonding. The bump diameter and array pitch are $10 \mu \mathrm{m}$ and $20 \mu \mathrm{m}$, respectively. substrate were successfully bonded, and no void or seam was observed between the bumps and the Au layer. Dieshear tests revealed a die-shear strength of more than 20 kgf, indicating that a sufficient bonding strength is achieved for this sample.

Flip-chip bonding was also carried out using test chips made from Au bump arrays with a bump diameter of 10 $\mu \mathrm{m}$, bump height of $10 \mu \mathrm{m}$, and array pitch of $30 \mu \mathrm{m}$. The chip size for these tests was $10 \mathrm{~mm} \times 10 \mathrm{~mm}$. Other conditions, such as pretreatment, were the same as those used in the above-mentioned investigations. Die-shear tests revealed a die-shear strength of more than $50 \mathrm{kgf}$, indicating that sufficient bonding strength is achieved.
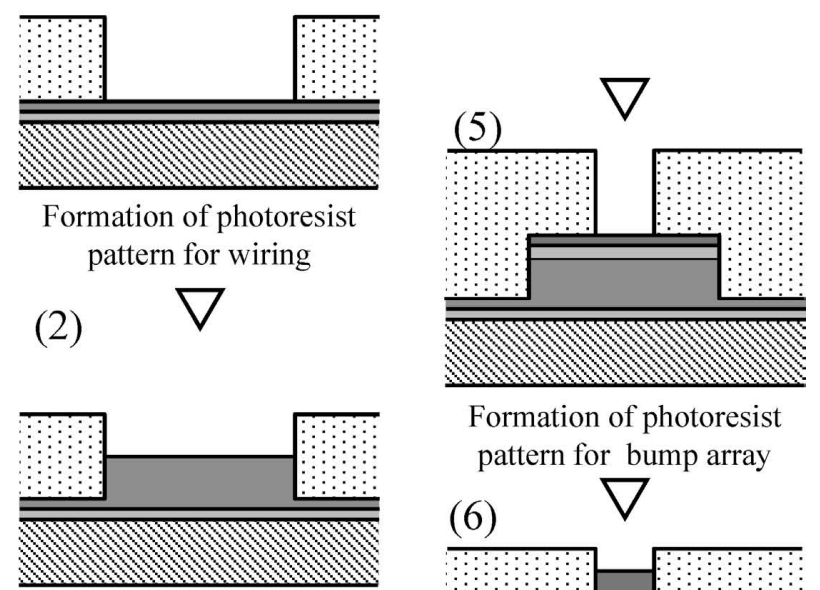

Formation of photoresist pattern for bump array

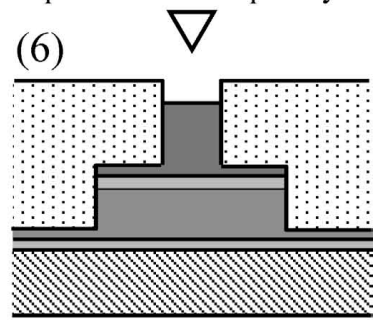

(3)
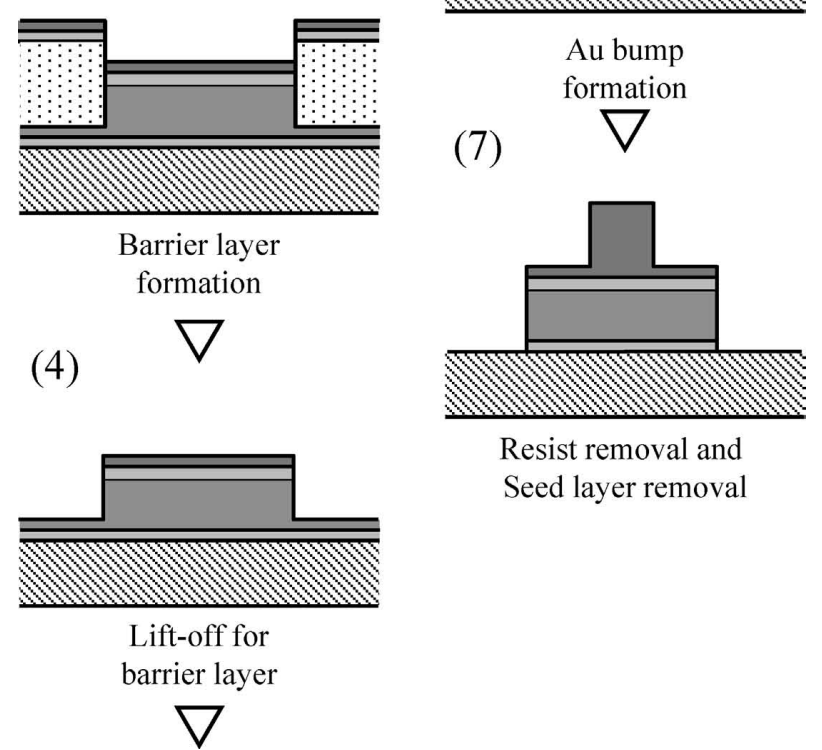

Resist removal and Seed layer removal

Fig. 7 Process flow for fabrication of a microbump array with wiring. 

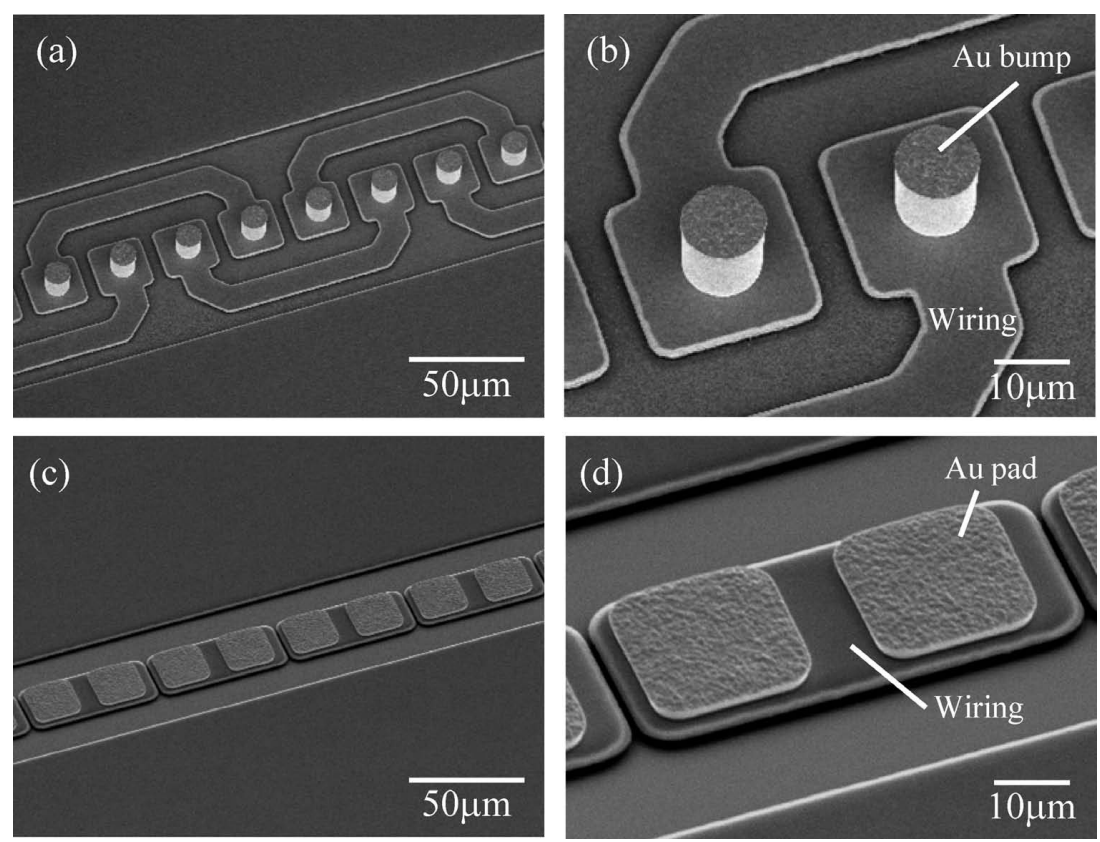

Fig. 8 SEM images of Au bump arrays on the chip (a), (b) and bump pad arrays on the substrate (c), (d). Barrier layer and seed layer were prepared by vacuum evaporation.

From these results, it appears that Au bump arrays fabricated with this method are suitable for fine-pitch, flip-chip bonding. Thus, the formation process using a non-cyanide plating bath proves practical for fabricating high-density interconnections consisting of ultra-fine pitch bump arrays.

\subsection{Fabrication of $\mathrm{Au}$ bumps on fine $\mathrm{Cu}$ wiring}

Figure 7 shows the process flow for fabricating $\mathrm{Au}$ microbump arrays with wiring. An evaporated $\mathrm{Cu}(100$ $\mathrm{nm}) / \mathrm{Ti}(10 \mathrm{~nm}) / \mathrm{Si}$ wafer was used as the substrate. First, the photoresist pattern for the wiring was prepared, with the thickness of the photoresist being $5 \mu \mathrm{m}$. Second, the $\mathrm{Cu}$ wiring, with a width of $22 \mu \mathrm{m}$ and thickness of $3 \mu \mathrm{m}$, was prepared by $\mathrm{Cu}$ electrodeposition. Third, a $\mathrm{Au}(50$ $\mathrm{nm}) / \mathrm{Ti}(50 \mathrm{~nm})$ layer was prepared by evaporation. The $\mathrm{Au}$ layer acts as a seed layer and the Ti layer acts as a barrier layer. Fourth, excess deposits of the barrier film were removed by the lift-off process using acetone. Fifth, the photoresist pattern for the bump array was formed, with the resist thickness and bump diameter being $12 \mu \mathrm{m}$ and $10 \mu \mathrm{m}$, respectively. Sixth, a Au bump array with a bump height of $10 \mu \mathrm{m}$ was prepared by electroless $\mathrm{Au}$ deposition. The final resist was removed, along with excess deposits of the seed layer/barrier layer. The $\mathrm{Cu}$ seed layer and $\mathrm{Ti}$ barrier layer were removed by chemical etching and reactive ion etching, respectively.

Figure 8 (a) and (b) show the Au micro bump array on a test chip. The array pitch is $30 \mu \mathrm{m}$. A smooth-walled Au bump array on $\mathrm{Cu}$ wiring was observed. Figure 8 (c) and

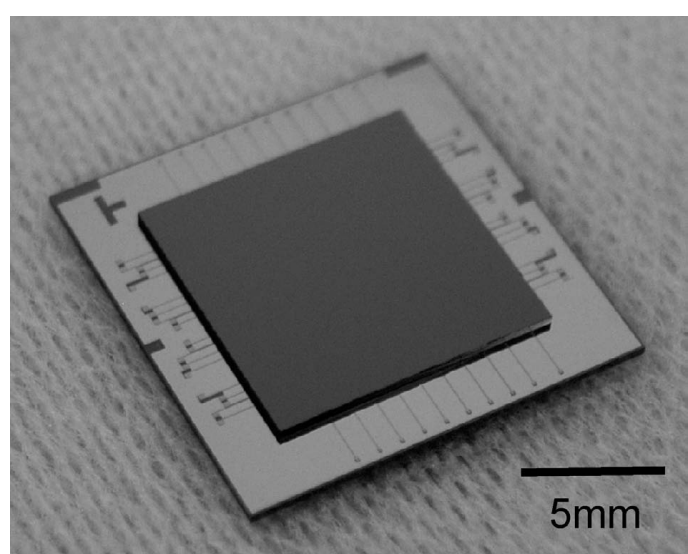

Fig. 9 Chip on substrate after flip-chip bonding.

(d) show Au micropads on the chip substrate. The test substrate was fabricated using the same process flow given in Fig. 7. A Au pad with dimensions of $20 \times 20 \mu \mathrm{m}^{2}$ and a thickness of $2 \mu \mathrm{m}$ was prepared for the bump connection by electroless Au deposition using Bath $\mathrm{K}$.

Using Au bump arrays fabricated by the methods discussed above, we performed chip-to-substrate flip-chip bonding, with the result shown in Fig. 9. The bonding strength is not as high as that in the previous investigation, but the electrical continuity test indicates that good electrical contact is established

\subsection{Fabrication of barrier/adhesive layer by electro- less deposition}

In the third step given in Fig. 7, the barrier layer was fab- 

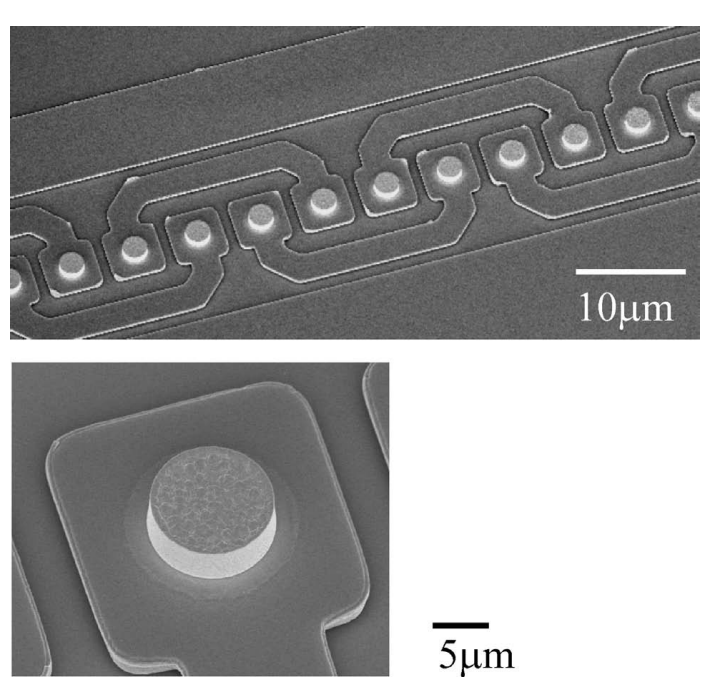

Figure10 SEM images of Au bump arrays on a chip. Both the barrier layer and the seed layer were prepared by electroless deposition.

ricated using a dry fabrication process. However, for wiring, all-wet fabrication processes were required. To overcome this, we developed an electroless deposition method for the barrier/adhesive layer.

Figure 10 shows the test chip made from Au bump arrays with a bump diameter of $10 \mu \mathrm{m}$, bump height of 5 $\mu \mathrm{m}$, and array pitch of $30 \mu \mathrm{m}$. The barrier layer was prepared by the semi-additive method using electroless $\mathrm{NiB}$ deposition, and films with thicknesses of $500 \mathrm{~nm}$ were produced. Using a $\mathrm{NiB}$ bath, we achieved selective deposition without activations (such as Pd activation). Before depositing Au bumps via electroless Au deposition, a Au seed layer was developed using displacement Au deposition. In this research, we used a non-cyanide Au plating bath. These results demonstrate the successful fabrication of $\mathrm{Au}$ bump arrays on $\mathrm{Cu}$ wiring using a non-cyanide process, and this process has high potential for achieving highdensity, flip-chip bonding.

\section{Conclusion}

We investigated the fabrication of Au microbump arrays deposited by non-cyanide electroless Au plating bath for flip-chip bonding. We developed an electroless Au deposition process to produce fine bump patterns with a high deposition rate using a non-cyanide bath. Using this new bath, we could fabricate Au bumps with a bump diameter of $10 \mu \mathrm{m}$ and bump height of $10 \mu \mathrm{m}$. Flip-chip bonding of the Au bump arrays thus produced resulted in good bonding strengths. Furthermore, we successfully deposited Au microbump arrays on $\mathrm{Cu}$ wiring. The practical feasibility of this technique was demonstrated by forming arrays with a bump height of $10 \mu \mathrm{m}$, bump diameter of $10 \mu \mathrm{m}$, and array pitch of $30 \mu \mathrm{m}$. The entire film formation process used a non-cyanide bath; thus, this process shows high potential for fabricating ultra-fine Au bump arrays. High density interconnects are a key requirement for Next-Generation Multi-Functional High-Density 3D Integration Technology.[7] This fabrication method is a candidate interconnect technology for the new 3D integration technology.

\section{References}

[1] J. U. Knickerbocker, P. S. Andry, L. P. Buchwalter, A. Deutsch, R. R. Horton, K. A. Jenkins, Y. H. Kwark, G. McVicker, C. S. Patel, R. J. Polastre, C. Schuster, A. Sharma, S. M. Sri-Jayantha, C. W. Surovic, C. K. Tsang, B. C. Webb, S. L. Wright, S. R. McKnight, E. J. Sprogis and B. Dang, "Development of next-generation system-on-package (SOP) technology based on silicon carriers with fine-pitch chip interconnection”, IBM J. Res. Develop., Vol. 49, No. 4-5, pp. 725-753, 2005.

[2] K. Takahashi, M. Umemoto, N. Tanaka, K. Tanida, Y. Nemoto, Y. Tomita, M. Tago and M. Bonkohara, "Ultra-high-density interconnection technology of three-dimensional packaging", Vol. 43, No. 8, pp. 1267-1279, 2003.

[3] K. Tanida, M. Umemoto, T. Morifuji, R. Kajiwara, Y. Tomita, N. Tanaka and K. Takahashi, "Au bump interconnection in $20 \mu \mathrm{m}$ pitch on 3D chip stacking technology,” Jpn. J. Appl. Phys., Vol. 42, No. 10, pp. 6390-6395, 2003.

[4] Y. Tomita, T. Torifuji, M. Tomisaka, S. Sumohara, Y. Nemoto, T. Sato, K. Takahashi and M. Bonkohara, "Cu bump interconnections in $20 \mu \mathrm{m}$-pitch at low temperature utilizing electroless tin-plating on 3D stacked LSI”, J. Chem. Eng. Jpn., Vol. 36, No. 2, pp. 199-125, 2003.

[5] A. Chinda, "Plating Technologies for Joins Used in Tape Carriers for Semiconductor Packages”, J. Surf. Finish. Soc. Jpn., Vol. 54, No. 2, pp. 118-123, 2003.

[6] H. Watanabe, "Cyanide-free Electroless Au plating processes for electronics devices”, pp. 272-275, "Advanced Technology for Surface Finishing", KantoGakuin Univ., Surface Eng. Res. Inst. Eds., Kogyo Chosakai Publishing Co., Ltd., 2006, (In Japanese).

[7] M. Aoyagi, "Prospect for Next-Generation Multi-Functional High-Density 3D Integration Technology”, J. Jpn. Inst. Electron. Packaging, Vol. 12, No. 4, pp. 301306, 2009, (In Japanese). 